\title{
Numerical Solutions of General Fourth Order Two Point Boundary Value Problems by Galerkin Method with Legendre Polynomials
}

\author{
Md. Bellal Hossain and Md. Shafiqul Islam*
}

Department of Mathematics, Dhaka University, Dhaka - 1000, Bangladesh

(Received: 20 April 2013; Accepted: 24 December 2013)

\begin{abstract}
In this paper, Galerkin weighted residual method is presented to find the numerical solutions of the general fourth order linear and nonlinear differential equations with essential boundary conditions. For this, the given differential equations and the boundary conditions over arbitrary finite domain $[a, b]$ are converted into its equivalent form over the interval $[0,1]$. Here the Legendre polynomials, over the interval $[0,1]$, are chosen as trial functions satisfying the corresponding homogeneous form of the Dirichlet boundary conditions. Details matrix formulations are derived for solving linear and nonlinear boundary value problems (BVPs). Numerical examples for both linear and nonlinear BVPs are considered to verify the proposed formulation and the results obtained are compared.
\end{abstract}

Keywords: Galerkin method, Fourth order linear and nonlinear BVPs, Legendre polynomials.

\section{Introduction}

Many higher order boundary value problems (BVPs) arise in the mathematical modeling of viscoelastic and inelastic flows, deformation of beams and plates deflection theory, beam element theory and many more applications of engineering and applied mathematics which are solved either analytically ${ }^{1}$ or numerically ${ }^{2,3}$. Only a limited number of such type of BVPs can be solved by using analytical methods leading to closed form solutions. For this, many researchers have attempted for the numerical solutions of fourth order BVPs to obtain high accuracy rapidly by using a numerous method such as, finite difference method, SincGalerkin method and also some other methods using polynomial and nonpolynomial spline functions. Among the various researchers, Usmani ${ }^{4}$ and Usmani and $\mathrm{Warsi}^{5}$ developed and analyzed second order and fourth order convergent methods for the solution of linear fourth order two-point boundary problem using quartic, quintic and sextic polynomial spline functions, respectively. Al-Said and Noor $^{6}$ and Al-Said et $a l^{7}$ demonstrated second order convergent method based on cubic and quartic polynomial spline functions for the solution of fourth order obstacle problems. Usmani ${ }^{8}$ established and discussed convergent second order and fourth order methods for this problem with the change in the boundary conditions for first order instead of second order derivatives using quintic and sextic polynomial spline functions, respectively. Also, Rashidinia and Golbabaee ${ }^{9}$, and Siddiqi and Akram $^{10}$ generated a deference scheme via quintic spline functions for this problem. Loghmani and Alavizadeh ${ }^{11}$ converted this problem into an optimal control problem and then constructed the approximate solution as a combination of quartic B-splines.

Van Daele et al $^{12}$ introduced a new second order method for solving the BVPs involving first derivatives based on nonpolynomial spline function. Siraj et al ${ }^{13}$ solved a system of third order boundary value problems using nonpolynomial spline functions. Ramadan et $a l^{14}$ proposed a second order convergent method for the numerical solution of second order BVP. Later Ramadan et $a l^{15}$ and Siraj-ulIslam et $a l^{16}$ have solved fourth order two-point boundary value problems using quintic nonpolynomial spline functions. Smith et $a^{17}$ solved only linear while El-Gamel et $\mathrm{al}^{18}$ solved the nonlinear BVPs by the technique of SincGalerkin method. Very recently Kasi et $a 1^{19}$ used Quintic Bsplines for solving fourth order BVP by the Galerkin method $^{3}$. Thus from the numerical survey, for fourth order BVPs, we note that a large number of authors have solved fourth order BVPs using spline functions which can be exploited easily but the numerical results converge slowly. Besides spline functions, there is another type of piecewise continuous polynomials, namely Legendre polynomials, which is available in the book ${ }^{2}$ may be used for numerical solutions of fourth order BVPs. Therefore, in this paper we try to present a simple Galerkin approach with Legendre polynomials as basis functions to solve the fourth order BVPs with essential boundary conditions.

However, in section II of this paper, a short description on Legendre polynomials is mentioned. In section III, the formulation of the Galerkin method with Legendre polynomials as basis functions are to be presented for solving linear fourth order BVP. Then we deduce the similar formulation for nonlinear problems in the next section with particular problems. The proposed formulation is verified on four linear and two nonlinear BVPs in section IV. Finally, in the last section, the conclusion of the paper is presented.

\section{Legendre Polynomials}

The general form of the Legendre polynomials [2] of degree $n$ is defined by

$$
L_{n}(x)=\sum_{r=0}^{N}(-1)^{r} \frac{(2 n-2 r) !}{2^{n} r !(n-r) !(n-2 r) !} x^{n-2 r}
$$

where $N=\frac{n}{2}$ for $n$ even and $N=\frac{n-1}{2}$ for $n$ odd.

The first few Legendre polynomials are given below:

$$
\begin{aligned}
& L_{1}(x)=x \\
& L_{2}(x)=\left(3 x^{2}-1\right) / 2 \\
& L_{3}(x)=\left(5 x^{3}-3 x\right) / 2 \\
& L_{4}(x)=\left(35 x^{4}-30 x^{2}+3\right) / 8 \\
& L_{5}(x)=\left(63 x^{5}-70 x^{3}+15 x\right) / 8
\end{aligned}
$$

*Author for correspondence e-mail: mdshafiqul_mat@du.ac.bd 
$L_{6}(x)=\left(231 x^{6}-315 x^{4}+105 x^{2}-5\right) / 16$

$L_{7}(x)=\left(429 x^{7}-693 x^{5}+315 x^{3}-35 x\right) / 16$

\section{Modified Legendre polynomials}

Here the Legendre polynomials are chosen such that they bijectively maps the interval $[0,1]$ to the interval [ implying that the polynomials are orthogonal on $[0,1]$.

The analogue of Rodrigues' formula for the Legendre polynomials is

$p_{n}(x)=\frac{1}{n !} \frac{d^{n}}{d x^{n}}\left(x^{2}-x\right)^{n}$.

To satisfy the condition $p_{n}(0)=p_{n}(1)=0, n \geq 1$, we modify the Legendre polynomials in Eq. (2) as

$p_{n}(x)=\left[\frac{1}{n !} \frac{d^{n}}{d x^{n}}\left(x^{2}-x\right)^{n}-(-1)^{n}\right](x-1)$.

Now from Eq. (3) we can write first few modified Legendre polynomials over the interval $[0,1]$ :

$$
\begin{aligned}
p_{1}(x)= & -2 x+2 x^{2} \\
p_{2}(x)= & 6 x-12 x^{2}+6 x^{3} \\
p_{3}(x)= & -12 x+42 x^{2}-50 x^{3}+20 x^{4} \\
p_{4}(x)= & 20 x-110 x^{2}+230 x^{3}-210 x^{4}+70 x^{5} \\
p_{5}(x)= & -30 x+240 x^{2}-770 x^{3}+1190 x^{4} \\
& -882 x^{5}+252 x^{6} \\
p_{6}(x)= & 42 x-462 x^{2}+2100 x^{3}-4830 x^{4} \\
& +5922 x^{5}-3696 x^{6}+924 x^{7} \\
p_{7}(x)= & -56 x+812 x^{2}-4956 x^{3}+15750 x^{4} \\
- & 28182 x^{5}+28644 x^{6}-15444 x^{7}+3432 x^{8} .
\end{aligned}
$$

Since modified Legendre polynomials have special properties at $x=0$ and $x=1: \quad p_{n}(0)=0 \quad$ and $p_{n}(1)=0, n \geq 1$ respectively, so that they can be used as set of basis function to satisfy the corresponding homogeneous form of the Dirichlet boundary conditions to derive the matrix formulation of fourth order BVP over the interval $[0,1]$.

\section{Matrix Formulation}

In this section we first obtain the rigorous formulation for fourth order linear BVP and then we extend our idea for solving nonlinear BVP. For this, we consider a general fourth order linear differential equation given by

$$
a_{4} \frac{d^{4} u}{d x^{4}}+a_{3} \frac{d^{3} u}{d x^{3}}+a_{2} \frac{d^{2} u}{d x^{2}}+a_{1} \frac{d u}{d x}+a_{0} u=r, a<x<b
$$

with Dirichlet boundary conditions

$$
u(a)=A_{0}, u(b)=B_{0}
$$

and the first derivative boundary conditions

$$
u^{\prime}(a)=A_{1}, u^{\prime}(b)=B_{1},
$$

where $A_{0}, A_{1}, B_{0}, B_{1}$ are finite real constants and $a_{4}, a_{3}, a_{2}, a_{1}, a_{0}$ and $r$ are all continuous functions of $x$ defined on the interval $[a, b]$. Since our aim is to use the Legendre polynomials as trial functions which are derived over the interval $[0,1]$, so the BVP, defined in Eqs. (4) (6), is to be converted to an equivalent problem on $[0,1]$ by replacing $x$ by $(b-a) x+a$, and thus we have

$$
c_{4} \frac{d^{4} u}{d x^{4}}+c_{3} \frac{d^{3} u}{d x^{3}}+c_{2} \frac{d^{2} u}{d x^{2}}+c_{1} \frac{d u}{d x}+c_{0} u=b, \quad 0<x<1,
$$

subject to the boundary conditions:

$$
\begin{aligned}
& u(0)=A_{0}, u(1)=B_{0}, \\
& \frac{1}{b-a} u^{\prime}(0)=A_{1}, \frac{1}{b-a} u^{\prime}(1)=B_{1},
\end{aligned}
$$

where

$$
\begin{aligned}
c_{4} & =\frac{a_{4}}{(b-a)^{4}}((b-a) x+a), \\
c_{3} & =\frac{a_{3}}{(b-a)^{3}}((b-a) x+a), \\
c_{2} & =\frac{a_{2}}{(b-a)^{2}}((b-a) x+a), \\
c_{1} & =\frac{a_{1}}{b-a}((b-a) x+a), \\
c_{0}= & a_{0}((b-a) x+a), b=r((b-a) x+a) .
\end{aligned}
$$

Using Legendre polynomials $p_{i}$, we assume an approximate solution in a form

$$
\tilde{u}=\theta_{0}+\sum_{i=1}^{n} a_{i} p_{i}
$$

Here $\theta_{0}$ is specified by the essential boundary conditions and $p_{i}(0)=p_{i}(1)=0$ for each $i=1,2, \ldots n$.

Using Eq. (10) into Eq. (7), the Galerkin weighted residual equations are

$$
\begin{array}{r}
\int_{0}^{1}\left[c_{4} \frac{d^{4} \tilde{u}}{d x^{4}}+c_{3} \frac{d^{3} \tilde{u}}{d x^{3}}+c_{2} \frac{d^{2} \tilde{u}}{d x^{2}}+c_{1} \frac{d \tilde{u}}{d x}+c_{0} \tilde{u}-b\right] \times p_{j} d x=0, \\
j=1,2, \ldots, n .
\end{array}
$$

Integrating by parts the terms up to second derivative on the left hand side of Eq. (11), we can obtain each term after applying the conditions prescribed in Eq. (8) as

$$
\int_{0}^{1} c_{4} \frac{d^{4} \tilde{u}}{d x^{4}} p_{j} d x=\left[c_{4} p_{j} \frac{d^{3} \tilde{u}}{d x^{3}}\right]_{0}^{1}-\int_{0}^{1} \frac{d}{d x}\left[c_{4} p_{j}\right] \frac{d^{3} \tilde{u}}{d x^{3}} d x
$$

$$
\text { [since } p_{j}(0)=p_{j}(1)=0 \text { ] }
$$




$$
\begin{gathered}
=-\left[\frac{d}{d x}\left[c_{4} p_{j}\right] \frac{d^{2} \tilde{u}}{d x^{2}}\right]_{0}^{1}+\int_{0}^{1} \frac{d^{2}}{d x^{2}}\left[c_{4} p_{j}\right] \frac{d^{2} \tilde{u}}{d x^{2}} d x \\
=-\left[\frac{d}{d x}\left[c_{4} p_{j}\right] \frac{d^{2} \tilde{u}}{d x^{2}}\right]_{0}^{1}+\left[\frac{d^{2}}{d x^{2}}\left[c_{4} p_{j}\right] \frac{d \tilde{u}}{d x}\right]_{0}^{1} \\
=-\left[\frac{d}{d x}\left[c_{4} p_{j}\right] \frac{d^{2} \tilde{u}}{d x^{2}}\right]_{0}^{1}+\left[\frac{d^{3}}{d x^{3}}\left[c_{4} p_{j}\right] \frac{d \tilde{u}}{d x} d x\right. \\
\left.-\left[\frac{d^{2}}{d x^{2}}\left[c_{4} p_{j}\right]\right]_{x=1} \times(b-a)\right]_{x=0} \times(b-a) A_{1}-\int_{0}^{1} \frac{d^{3}}{d x^{3}}\left[c_{4} p_{j}\right] \frac{d \tilde{u}}{d x} d x
\end{gathered}
$$

$$
\begin{aligned}
& \int_{0}^{1} c_{3} \frac{d^{3} \tilde{u}}{d x^{3}} p_{j} d x \\
& =\left[c_{3} p_{j} \frac{d^{2} \tilde{u}}{d x^{2}}\right]_{0}^{1}-\int_{0}^{1} \frac{d}{d x}\left[c_{3} p_{j}\right] \frac{d^{2} \tilde{u}}{d x^{2}} d x \\
& =-\left[\frac{d}{d x}\left[c_{3} p_{j}\right]\right]_{x=1} \times(b-a) B_{1}+\left[\frac{d}{d x}\left[c_{3} p_{j}\right]\right]_{x=0} \\
& \quad \times(b-a) A_{1}+\int_{0}^{1} \frac{d^{2}}{d x^{2}}\left[c_{3} p_{j}\right] \frac{d \tilde{u}}{d x} d x
\end{aligned}
$$

$$
\begin{gathered}
\int_{0}^{1} c_{2} \frac{d^{2} \tilde{u}}{d x^{2}} p_{j} d x \\
=\left[c_{2} p_{j} \frac{d \tilde{u}}{d x}\right]_{0}^{1}-\int_{0}^{1} \frac{d}{d x}\left[c_{2} p_{j}\right] \frac{d \tilde{u}}{d x} d x \\
=-\int_{0}^{1} \frac{d}{d x}\left[c_{2} p_{j}\right] \frac{d \tilde{u}}{d x} d x
\end{gathered}
$$

Substituting Eqs. (12) - (14) into Eq. (11) and using approximation for $\tilde{u}(x)$ given in Eq. (10), and after rearranging the terms for the resulting equations, we get a system of equations in the matrix form as

$$
\sum_{i=1}^{n} K_{i, j} a_{i}=F_{j}, \quad j=1,2, \ldots, n .
$$

where

$$
\begin{aligned}
K_{i, j}= & \int_{0}^{1}\left\{\left[-\frac{d^{3}}{d x^{3}}\left[c_{4} p_{j}\right]+\frac{d^{2}}{d x^{2}}\left[c_{3} p_{j}\right]\right]\right. \\
& \left.\left.-\frac{d}{d x}\left[c_{2} p_{j}\right]+c_{1} p_{j}\right] \frac{d p_{i}}{d x}+c_{0} p_{i} p_{j}\right\} d x
\end{aligned}
$$

$$
\begin{aligned}
F_{j} & =\int_{0}^{1}\left\{s p_{j}+\left[\frac{d^{3}}{d x^{3}}\left[c_{4} p_{j}\right]-\frac{d^{2}}{d x^{2}}\left[c_{3} p_{j}\right]\right.\right. \\
+ & \left.\left.\frac{d}{d x}\left[c_{2} p_{j}\right]-c_{1} p_{j}\right] \frac{d \theta_{0}}{d x}+-c_{0} p_{j} \theta_{0}\right\} d x \\
+ & {\left[\frac{d}{d x}\left[c_{3} p_{j}\right]-\frac{d^{2}}{d x^{2}}\left[c_{4} p_{j}\right]\right]_{x=1} \times(b-a) B_{1} } \\
- & {\left[\frac{d}{d x}\left[c_{3} p_{j}\right]-\frac{d^{2}}{d x^{2}}\left[c_{4} p_{j}\right]\right]_{x=0} \times(b-a) A_{1} } \\
& +\left[\frac{d}{d x}\left[c_{4} p_{j}\right] \frac{d^{2} \theta_{0}}{d x^{2}}\right]_{x=1}-\left[\frac{d}{d x}\left[c_{4} p_{j}\right] \frac{d^{2} \theta_{0}}{d x^{2}}\right]_{x=0} .
\end{aligned}
$$

Solving the system of equations specified by Eq. (15), we find the values of the parameters $a_{i}$, and then substituting these parameters into Eq. (10), we get the approximate solution of the BVP defined in Eqs. (7) - (9). If we replace $x$ by $\frac{x-a}{b-a}$ in $\tilde{u}$, then we get the desired approximate solution of the BVP defined in Eqs. (4) - (6).

For nonlinear fourth order BVP, we first compute the initial values neglecting the nonlinear terms and use the system (15). Then using the iterative method we find the numerical approximations for desired nonlinear BVP. This formulation is described through the numerical examples in the next section.

\section{Numerical Results}

To test the applicability of the proposed method, we consider two linear and two nonlinear problems with boundary conditions of the type (5) and (6) because the exact solutions for these problems are available in the literature. For all the examples, the solutions obtained by the proposed method are compared with the exact solutions. All the computations are performed using MATLAB. The convergence of linear BVP is calculated by

$$
E=\left|\tilde{u}_{n+1}(x)-\tilde{u}_{n}(x)\right|<\delta,
$$

where $\tilde{u}_{n}(x)$ denotes the approximate solution using $n$ polynomials and $\delta$ depends on the problem which varies from $10^{-7}$ to $10^{-10}$. In addition, the convergence of nonlinear BVP is assumed when the absolute error of two consecutive iterations, $\delta$ satisfies

$$
\left|\tilde{u}_{n}^{N+1}-\tilde{u}_{n}^{N}\right|<\delta
$$


where $N$ is the Newton's iteration number and $\delta$ varies from $10^{-11}$ to $10^{-13}$.

Example 1. Consider the linear $\mathrm{BVP}^{9,16,19}$

$$
\frac{d^{4} u}{d x^{4}}+4 u=1,-1<x<1
$$

subject to the boundary conditions

$$
u(-1)=u(1)=0, u^{\prime}(-1)=-u^{\prime}(1)=\frac{\sinh 2-\sin 2}{4(\cosh 2+\cos 2)},
$$

whose exact solution is,

$$
\begin{aligned}
u(x)= & \frac{1}{4}-\frac{1}{2}(\sinh 1 \sin 1 \sinh x \sin x \\
& +\cosh 1 \cos 1 \cosh x \cos x) /(\cosh 2+\cos 2) .
\end{aligned}
$$

The equivalent BVP over $[0,1]$ to the BVP defined in Eqs. (18) and (19) is,

$$
\frac{1}{2^{4}} \frac{d^{4} u}{d x^{4}}+4 u=1, \quad 0<x<1
$$

subject to the boundary conditions

$$
\begin{aligned}
& u(0)=u(1)=0, \\
& \frac{1}{2} u^{\prime}(0)=-\frac{1}{2} u^{\prime}(1)=\frac{\sinh 2-\sin 2}{4(\cosh 2+\cos 2)} .
\end{aligned}
$$

Using the method illustrated in section III,

$$
\sum_{i=1}^{n} K_{i, j} a_{i}=F_{j}, j=1,2, \ldots, n
$$

where

$$
\begin{gathered}
K_{i, j}=\int_{0}^{1}\left\{-\frac{d^{3} p_{j}}{d x^{3}} \frac{d p_{i}}{d x}+64 p_{i} p_{j}\right\} d x \\
-\left[\frac{d p_{j}}{d x} \frac{d^{2} p_{i}}{d x^{2}}\right]_{x=1}+\left[\frac{d p_{j}}{d x} \frac{d^{2} p_{i}}{d x^{2}}\right]_{x=0} \\
F_{j}=\int_{0}^{1} 16 p_{j} d x+\left[\frac{d^{2} p_{j}}{d x^{2}}\right]_{x=1}\left\{\frac{\sinh 2-\sin 2}{2(\cosh 2+\cos 2)}\right\} \\
+\left[\frac{d^{2} p_{j}}{d x^{2}}\right]_{x=0}\left\{\frac{\sinh 2-\sin 2}{2(\cosh 2+\cos 2)}\right\}
\end{gathered}
$$

Table 1. Numerical results of example 1.

\begin{tabular}{c|c|c|c}
\hline$x$ & Exact Solutions & $\begin{array}{c}\text { Proposed method } \\
\text { using 11 } \\
\text { polynomials }\end{array}$ & Absolute Error \\
\hline-1.0 & 0.0000000000 & 0.0000000000 & 0.000000000000 \\
-0.8 & 0.0397692607 & 0.0397692607 & $4.6837534 \mathrm{E}-015$ \\
-0.6 & 0.0749849828 & 0.0749849828 & $1.7749691 \mathrm{E}-014$ \\
-0.4 & 0.1023106409 & 0.1023106409 & $7.2997164 \mathrm{E}-015$ \\
-0.2 & 0.1195382314 & 0.1195382314 & $7.9422580 \mathrm{E}-014$ \\
0.0 & 0.1254157424 & 0.1254157424 & $1.3106183 \mathrm{E}-016$ \\
0.2 & 0.1195382314 & 0.1195382314 & $9.5423669 \mathrm{E}-014$ \\
0.4 & 0.1023106409 & 0.1023106409 & $1.5751289 \mathrm{E}-014$ \\
0.6 & 0.0749849828 & 0.0749849828 & $2.3370195 \mathrm{E}-014$ \\
0.8 & 0.0397692607 & 0.0397692607 & $3.1780134 \mathrm{E}-015$ \\
1.0 & 0.0000000000 & 0.0000000000 & 0.000000000000 \\
\hline
\end{tabular}

Solving the system in Eq. (22), we obtain the values of the parameters and then substituting these parameters into the similar form of Eq. (10), we get the approximate solution to the BVP, defined in Eqs. (20) and (21), for different values of $n$. If we replace $x$ by $\frac{x+1}{2}$ in $\tilde{u}(x)$, then we get the desired approximate solution of the BVP defined in Eqs. (18) and (19). The numerical results for this problem are shown in Table 1 . The maximum absolute error obtained by the proposed method is $9.542 \times 10^{-14}$. On the contrary, the errors have been obtained by Rashidinia and Golbabaee ${ }^{9}$, Siraj-ul-Islam et $a l^{16}$ and Kasi et $a l^{19}$ are $6.22 \times 10^{-6}$, $2.82 \times 10^{-12}$ and $1.303 \times 10^{-6}$, respectively.

Example 2. Consider the linear $\mathrm{BVP}^{5,9,19}$

$$
\frac{d^{4} u}{d x^{4}}+x u=-\left(8+7 x+x^{3}\right) e^{x}, \quad 0<x<1
$$

subject to the boundary conditions

$$
u(0)=u(1)=0, u^{\prime}(0)=1, u^{\prime}(1)=-e .
$$

whose exact solution is $u(x)=x(1-x) e^{x}$.

Applying the proposed method, mentioned in section III, the numerical results for this problem are given in Table 2. The maximum absolute error obtained by the proposed method is $2.502 \times 10^{-13}$. It is observed that using splines the maximum errors have been found by Al-Said et $a l^{5}$, Rashidinia and Golbabaee $^{9}$, and Kasi et $a l^{19}$ are upto $2.36 \times 10^{-7}$, $5.37 \times 10^{-6}$ and $5.99 \times 10^{-6}$, respectively.

Table 2. Numerical results of example 2.

\begin{tabular}{c|c|c|c}
\hline$x$ & Exact Solutions & $\begin{array}{c}\text { Proposed } \\
\text { method using 11 } \\
\text { polynomials }\end{array}$ & Absolute Error \\
\hline 0.0 & 0.0000000000 & 0.0000000000 & 0.000000000000 \\
0.1 & 0.0994653826 & 0.0994653826 & $4.6490589 \mathrm{E}-014$ \\
0.2 & 0.1954244413 & 0.1954244413 & $1.2767565 \mathrm{E}-013$ \\
0.3 & 0.2834703496 & 0.2834703496 & $1.7824631 \mathrm{E}-013$ \\
0.4 & 0.3580379274 & 0.3580379274 & $2.8310687 \mathrm{E}-015$ \\
0.5 & 0.4121803177 & 0.4121803177 & $2.5018876 \mathrm{E}-013$ \\
0.6 & 0.4373085121 & 0.4373085121 & $1.8657298 \mathrm{E}-013$ \\
0.7 & 0.4228880686 & 0.4228880686 & $8.5431662 \mathrm{E}-014$ \\
0.8 & 0.3560865486 & 0.3560865486 & $2.0078383 \mathrm{E}-013$ \\
0.9 & 0.2213642800 & 0.2213642800 & $1.4432899 \mathrm{E}-013$ \\
1.0 & 0.0000000000 & 0.0000000000 & 0.000000000000 \\
\hline
\end{tabular}

Example 3. Consider the nonlinear $\mathrm{BVP}^{19}$

$$
\frac{d^{4} u}{d x^{4}}=\sin x+\sin ^{2} x-\left(\frac{d^{2} u}{d x^{2}}\right)^{2}, 0<x<1
$$

subject to boundary conditions:

$$
u(0)=0, u(1)=\sin 1, u^{\prime}(0)=1, u^{\prime}(1)=\cos 1 .
$$

The exact solution of this BVP is $u(x)=\sin x$.

Consider the approximate solution of $u(x)$ as

$$
\tilde{u}=\theta_{0}+\sum_{i=1}^{n} a_{i} p_{i}, n \geq 1 .
$$


Here $\theta_{0}=x \sin 1$ is specified by the essential boundary conditions in Eq. (28). Also $p_{i}(0)=p_{i}(1)=0$ for each $i=1,2, \ldots, n$.

Using Eq. (29) into Eq. (27), the Galerkin weighted residual equation is

$$
\int_{0}^{1}\left[\frac{d^{4} \tilde{u}}{d x^{4}}+\left(\frac{d^{2} \tilde{u}}{d x^{2}}\right)^{2}-\sin x-\sin ^{2} x\right] p_{k} d x=0
$$

Integrating first term of Eq. (30) by parts, we obtain

$$
\begin{aligned}
& \int_{0}^{1} \frac{d^{4} \tilde{u}}{d x^{4}} p_{k} d x=\left[p_{k} \frac{d^{3} \tilde{u}}{d x^{3}}\right]_{0}^{1}-\int_{0}^{1} \frac{d p_{k}}{d x} \frac{d^{3} \tilde{u}}{d x^{3}} d x \\
& =-\left[\frac{d p_{k}}{d x} \frac{d^{2} \tilde{u}}{d x^{2}}\right]_{0}^{1}+\int_{0}^{1} \frac{d^{2} p_{k}}{d x^{2}} \frac{d^{2} \tilde{u}}{d x^{2}} d x \\
& =-\left[\frac{d p_{k}}{d x} \frac{d^{2} \tilde{u}}{d x^{2}}\right]_{0}^{1}+\left[\frac{d^{2} p_{k}}{d x^{2}} \frac{d \tilde{u}}{d x}\right]_{0}^{1}-\int_{0}^{1} \frac{d^{3} p_{k}}{d x^{3}} \frac{d \tilde{u}}{d x} d x \\
& =-\left[\frac{d p_{k}}{d x}\right]_{x=1}\left[\theta_{0}^{\prime \prime}+\sum_{i=1}^{n} a_{i} p_{i}^{\prime \prime}\right]_{x=1}+\left[\frac{d p_{k}}{d x}\right]_{x=0}\left[\theta_{0}^{\prime \prime}+\sum_{i=1}^{n} a_{i} p_{i}^{\prime \prime}\right]_{x=0} \\
& +\left[\frac{d^{2} p_{k}}{d x^{2}}\right]_{x=1} \times \cos 1-\left[\frac{d^{2} p_{k}}{d x^{2}}\right]_{x=0} \quad-\int_{0}^{1} \frac{d^{3} p_{k}}{d x^{3}}\left[\theta_{0}^{\prime}+\sum_{i=0}^{1} a_{i} p_{i}^{\prime}\right]_{d x}
\end{aligned}
$$

Putting Eq. (31) into Eq. (30) we obtain the equivalent matrix form

$$
(D+B) A=G,
$$

where the elements of $A, B, D, G$ are $a_{i}, b_{i, k}, d_{i, k}$ and $g_{k}$ respectively, given by

$$
\begin{gathered}
d_{i, k}=\int_{0}^{1}\left[-\frac{d^{3} p_{k}}{d x^{3}} \frac{d p_{i}}{d x}+2 \frac{d^{2} \theta_{0}}{d x^{2}} \frac{d^{2} p_{i}}{d x^{2}} p_{k}\right] d x \\
-\left[\frac{d p_{k}}{d x} \frac{d^{2} p_{i}}{d x^{2}}\right]_{x=1}+\left[\frac{d p_{k}}{d x} \frac{d^{2} p_{i}}{d x^{2}}\right]_{x=0} \\
g_{k}=\int_{0}^{1}\left[\left(\sin x+\sin ^{2} x\right) p_{k}+\frac{d^{3} p_{k}}{d x^{3}} \frac{d \theta_{0}}{d x}-\left(\frac{d^{2} \theta_{0}}{d x^{2}}\right)^{2} p_{k}\right] d x \\
+\left[\frac{d p_{k}}{d x} \frac{d^{2} \theta_{0}}{d x^{2}}\right]_{x=1}^{n} a_{j}\left(\frac{d^{2} p_{i}}{d x^{2}} \frac{d^{2} p_{j}}{d x^{2}} p_{k}\right) d x \\
{\left[\frac{d p_{k}}{d x} \frac{d^{2} \theta_{0}}{d x^{2}}\right]_{x=0}}
\end{gathered}
$$

$$
-\left[\frac{d^{2} p_{k}}{d x^{2}}\right]_{x=1} \times \cos 1+\left[\frac{d^{2} p_{k}}{d x^{2}}\right]_{x=0}
$$

The initial values of these coefficients $a_{i}$ are obtained by applying Galerkin method to the BVP neglecting the nonlinear terms in Eq. (27). That is, to find initial coefficients we will solve the system

$$
D A=G
$$

whose matrices are constructed from

$$
\begin{aligned}
& d_{i, k}= \int_{0}^{1}\left[-\frac{d^{3} p_{k}}{d x^{3}} \frac{d p_{i}}{d x}\right] d x-\left[\frac{d p_{k}}{d x} \frac{d^{2} p_{i}}{d x^{2}}\right]_{x=1} \\
&+\left[\frac{d p_{k}}{d x} \frac{d^{2} p_{i}}{d x^{2}}\right]_{x=0} \\
& g_{k}= \int_{0}^{1}\left[\left(\sin x+\sin ^{2} x\right) p_{k}+\frac{d^{3} p_{k}}{d x^{3}} \frac{d \theta_{0}}{d x}\right] d x \\
&+\left.+\frac{d p_{k}}{d x} \frac{d^{2} \theta_{0}}{d x^{2}}\right]_{x=1}-\left[\frac{d p_{k}}{d x} \frac{d^{2} \theta_{0}}{d x^{2}}\right]_{x=0} \\
&-\left[\frac{d^{2} p_{k}}{d x^{2}}\right]_{x=1} \times \cos 1+\left[\frac{d^{2} p_{k}}{d x^{2}}\right]_{x=0}
\end{aligned}
$$

Table 3. Numerical results of example 3.

\begin{tabular}{c|c|c|c}
\hline$x$ & Exact Solutions & $\begin{array}{c}\text { Proposed method } \\
\text { using } 8 \\
\text { polynomials }\end{array}$ & Absolute Error \\
\hline 0.0 & 0.0000000000 & 0.0000000000 & 0.00000000000 \\
0.1 & 0.0998334166 & 0.0998334165 & $1.573325 \mathrm{E}-010$ \\
0.2 & 0.1986693308 & 0.1986693307 & $1.140406 \mathrm{E}-010$ \\
0.3 & 0.2955202067 & 0.2955202065 & $1.492606 \mathrm{E}-010$ \\
0.4 & 0.3894183423 & 0.3894183422 & $9.988721 \mathrm{E}-011$ \\
0.5 & 0.4794255386 & 0.4794255385 & $6.413814 \mathrm{E}-011$ \\
0.6 & 0.5646424734 & 0.5646424733 & $7.907730 \mathrm{E}-011$ \\
0.7 & 0.6442176872 & 0.6442176872 & $6.589806 \mathrm{E}-011$ \\
0.8 & 0.7173560909 & 0.7173560909 & $2.166500 \mathrm{E}-011$ \\
0.9 & 0.7833269096 & 0.7833269096 & $1.818679 \mathrm{E}-011$ \\
1.0 & 0.8414709848 & 0.8414709848 & 0.00000000000 \\
\hline
\end{tabular}

Once the initial values of the $a_{i}$ are obtained from Eq. (36), they are substituted into Eq. (32) to obtain new estimates for the values of $a_{i}$. This iteration process continues until the converged values of the unknown parameters are obtained. Substituting the final values of the parameters into Eq. (29), we obtain an approximate solution of the BVP defined in Eqs. (27) and (28). The numerical results for this problem are presented in Table 3. The maximum absolute error obtained by the proposed method is $1.573 \times 10^{-10}$ using 5 iterations. On the other hand, the maximum absolute error has been obtained by Kasi et $a l^{19}$ is $1.359 \times 10^{-5}$. 
Example 4. Consider the nonlinear $\mathrm{BVP}^{18,19}$

$$
\frac{d^{4} u}{d x^{4}}-6 e^{-4 u}=-12(1+x)^{-4}, 0<x<1
$$

subject to the boundary conditions:

$$
u(0)=0, u(1)=\ln 2, \quad u^{\prime}(0)=1, u^{\prime}(1)=0.5 .
$$

The exact solution is $u(x)=\ln (1+x)$.

Following the proposed method illustrated as in example 3 the numerical results for this problem are summarized in Table 4. The maximum absolute error obtained by the present method is $4.075 \times 10^{-8}$ using 5 iterations. The maximum absolute error has been obtained so far by ElGamel et al ${ }^{18}$ and Kasi et al ${ }^{19}$ are $2.2 \times 10^{-8}$ and $4.917 \times 10^{-6}$ respectively.

Table 4. Numerical results of example 4.

\begin{tabular}{c|c|c|c}
\hline$x$ & Exact Solutions & $\begin{array}{c}\text { Proposed method } \\
\text { using 9 polynomials }\end{array}$ & Absolute error \\
\hline 0.0 & 0.0000000000 & 0.0000000000 & 0.00000000000 \\
0.1 & 0.0953101798 & 0.0953101410 & $3.883659 \mathrm{E}-008$ \\
0.2 & 0.1823215568 & 0.1823215160 & $4.074934 \mathrm{E}-008$ \\
0.3 & 0.2623642645 & 0.2623642240 & $4.050873 \mathrm{E}-008$ \\
0.4 & 0.3364722366 & 0.3364722108 & $2.582027 \mathrm{E}-008$ \\
0.5 & 0.4054651081 & 0.4054650836 & $2.446640 \mathrm{E}-008$ \\
0.6 & 0.4700036292 & 0.4700036072 & $2.206729 \mathrm{E}-008$ \\
0.7 & 0.5306282511 & 0.5306282371 & $1.392392 \mathrm{E}-008$ \\
0.8 & 0.5877866649 & 0.5877866546 & $1.032887 \mathrm{E}-008$ \\
0.9 & 0.6418538862 & 0.6418538815 & $4.673076 \mathrm{E}-009$ \\
1.0 & 0.6931471806 & 0.6931471806 & 0.00000000000 \\
\hline
\end{tabular}

\section{Conclusion}

We have presented a simple and accurate method to solve a fourth order BVPs. We have extended a Galerkin method with Legendre polynomials as basis functions for solving fourth order BVPs with essential boundary conditions. The concentration has given not only on the performance of the results but also on the formulation. The proposed method is applied to solve a several number of linear and nonlinear problems to test the efficiency of the method. The numerical results obtained by the proposed method are in good agreement with the exact solutions and also confirm with great accuracy than the results obtained by the previous methods so far. In addition, to the knowledge of the present authors', none has attempted to solve the fourth order BVP using Legendre polynomials. Further we wish to extend our idea for solving any higher even order BVPs by the proposed method.

\section{Acknowledgement}

The authors acknowledge, with thanks, the comments of their referee on the first version of the paper. The first author is grateful to the Ministry of Science, Information and Communication Technology (ICT), Bangladesh for granting Bangabandhu fellowship and also the authority of the Patuakhali Science and Technology University (PSTU) for study leave during the period of research work.

\section{References}

1. Boyce, W. E. and R. C. DiPrima, 2011. Elementary Differential Equations and Boundary Value Problems, Wiley India (P) Ltd., 665-673.

2. Atkinson, K. E., 1989. An Introduction to Numerical Analysis, John Wiley \& Sons, NY, ${ }^{\text {nd }}$ Edition, 210-215.

3. Lewis, P. E. and J. P. Ward, 1991. The Finite Element Method, Principles and Applications, Addison-Wesley, 26-41.

4. Usmani, R. A. 1992. The use of quartic splines in the numerical solution of a fourth-order boundary value problems. J Comput Appl Math, 44, 187 - 99.

5. Usmani, R. A. and S. A. Warsi, 1980. Smooth spline solutions for boundary value problems in plate deflection theory, Comp Math Appl, 6, 205-11.

6. Al-Said, E. A. and M. A. Noor, 2002. Quartic spline method for solving fourth order obstacle boundary value problems. $J$ Comput Appl Math, 143,107 - 16.

7. Al-Said, E. A. and M. A. Noor, T. M. Rassias, 2006. Cubic splines method for solving fourth-order obstacle boundary value problems, Appl Math Comput, 174, 180 - 187.

8. Usmani, R. A., 1980. Smooth spline approximations for the solution of a boundary value problem with engineering applications, J Comput Appl Math, 6, 93 - 98.

9. Rashidinia, J. and A. Golbabaee, 2005. Convergence of numerical solution of a fourth-order boundary value problem, Appl Math Comput, 171, 1296 - 1305.

10. Siddiqi, S. S. and G. Akram, 2007. Solution of the system of fourth order boundary value problems using nonpolynomial spline technique, Appl Math Comput, 185, 128-135.

11. Loghmani, G. B. and S. R. Alavizadeh, 2007. Numerical solution of fourth-order problems with separated boundary conditions, Appl Math Comput, 191, 571 - 581.

12. Van Daele, M., G. Vanden Berghe and H. De Meyer, 1994. A smooth approximation for the solution of a fourth order boundary value problem based on nonpolynomial splines, $J$ Comput Appl Math, 51, 383 - 394.

13. Siraj-ul-Islam, M. A. Khan and I. A. Tirmizi, E. H. Twizell, 2005. Nonpolynomial spline approach to the solution of a system of third-order boundary value problems, Appl Math Comput, 168,152 - 163.

14. Ramadan, M. A., I. F. Lashien and W. K. Zahra, 2007. Polynomial and non-polynomial spline approaches to the numerical solution of second order boundary value problems, Appl Math Comput, 184, 476 - 484.

15. Ramadan, M. A., I. F. Lashien and W. K. Zahra, 2009. Quintic nonpolynomial spline solutions for fourth order twopoint boundary value problem, Commun. Nonlinear Sc. Numer. Simul, 14, 1105-1114.

16. Siraj-ul-Islam, I. A. Tirmizi and S. Ashraf, 2006. A class of methods based on non-polynomial spline functions for the solution of a special fourth order boundary value problems with engineering applications, Appl. Math. Comput, 174, 1169-1180.

17. Smith, R. C., G. A. Bogar, K. L. Bowers and J. Lund, 1991. The Sinc-Galerkin method for fourth-order differential equations, Siam J. Numer. Anal., 28, 760 - 788.

18. El-Gamel, M., S. H. Behiry and H. Hashish, 2003. Numerical method for the solution of special nonlinear fourth-order boundary value problems, Appl. Math. Comput. 145717 734.

19. Kasi, V. K. N. S., P. M. Krishna and S. R. Koneru, 2010. Numerical solutions of fourth order boundary value problems by Galerkin method with Quintic B-splines, Int. Jn Nonlinear Sc., 10, $222-230$. 
Dhaka Univ. J. Sci. 62(2): 103-108, 2014 ((July) 